\title{
Importância da experiência clínica para a mensuração da curva escoliótica de crianças pela técnica de Cobb
}

\author{
The importance of clinical experience for the measurement of scoliosis curve in children by \\ Cobb technique
}

\section{La importancia de la experiencia clinica en la medición de la curva escoliótica de niños mediante la técnica de Cobb}

\section{Bárbara Vendramini Marchetti', Eduardo Raupp², Juliana Adami Sedrez ${ }^{3}$, Rafael Paiva Ribeiro4, Cláudia Tarragô Candotti ${ }^{5}$}

RESUMO I A escoliose é definida como uma deformidade com desvio lateral da coluna no plano coronal, torsão da coluna e do tronco e distúrbio no perfil sagital. Essa alteração postural é avaliada por meio de radiografia de incidência anteroposterior, utilizando-se o método de Cobb. O objetivo do estudo é verificar a influência da experiência do avaliador sobre a confiabilidade intraexaminador e interexaminador do ângulo Cobb em curvaturas escolióticas de crianças. Foram incluídas na pesquisa 39 crianças portadoras de escoliose idiopática, com idade entre 7 e 18 anos. Os exames foram avaliados por dois fisioterapeutas, um quiropraxista e um estudante de fisioterapia - cada um avaliando duas vezes cada exame. A segunda avaliação ocorreu após sete dias, para confiabilidade intraexaminador. Ademais, as primeiras avaliações forneceram dados para confiabilidade interexaminador. A análise estatística foi realizada com coeficiente de correlação intraclasse (CCl), análise de Bland e Altman e análise descritiva do desvio absoluto médio, erro-padrão de medição e mínima mudança detectável. Observou-se boa confiabilidade $(\mathrm{CCl}>0,5)$ para as análises intraexaminadores entre os profissionais, e confiabilidade fraca $(\mathrm{CCl}=0,4)$ para $\mathrm{O}$ avaliador inexperiente. A confiabilidade interexaminador dos profissionais foi boa $(\mathrm{CCl}=0,6)$, e com a presença do avaliador inexperiente foi fraca $(\mathrm{CCl}=0,3)$. As avaliações entre os profissionais apresentaram menor variabilidade das medidas e valores de desvio-padrão quando comparadas com as do avaliador inexperiente. A mensuração dos ângulos da escoliose por meio do método de Cobb realizada por profissionais experientes apresentou melhores índices de concordância e de confiabilidade intra e interexaminadores e menor desvio-padrão e variabilidade entre as medidas.

Descritores | Coluna Vertebral; Raios X; Reprodutibilidade dos Testes.

ABSTRACT I Scoliosis is defined as a deformity with lateral
deviation of the spine in the coronal plane, torsion of the
spine and trunk, and disturbances in the sagittal profile. This
postural alteration is evaluated by anteroposterior incidence
radiography using the Cobb method. The objective of this
study was to verify the influence of evaluator experience
on inter- and intra-rater reliability of the Cobb angle of
scoliosis curvatures in children. In total, 39 patients aged
7 to 18 years with idiopathic scoliosis were included in
this study. The exams were evaluated by two physical
therapists, a chiropractor and a physical therapy student.
Each evaluator rated each exam twice and the second
evaluation occurred after seven days, characterizing the
intra-rater reliability. Furthermore, the first evaluations
provided the inter-rater reliability. Statistical analysis was
performed with intraclass correlation coefficient (ICC),
Bland-Altman analysis, descriptive analysis of mean absolute
deviation, standard error of measurement, and minimum 
detectable chance. Correlations ranged from good (ICC>0.5) for intra-rater reliability among professionals to weak (ICC=0.4) for the inexperienced evaluator. The inter-rater reliability of the professional's evaluations was good (ICC=0.6) and the same analysis with the presence of an inexperienced evaluator was weak (ICC=0.3). Evaluations among professionals showed less variability of measurements and standard deviation values compared to the inexperienced evaluator. The measurement of the angles of the scoliosis through the Cobb method carried out by experienced professionals showed better agreement as well as intra- and interrater reliability, lower standard deviation, and variability among the measurements.

Keywords | Spine; X-Rays; Reproducibility of Results.

RESUMEN I La escoliosis se define como una alteración con curvatura lateral de la columna vertebral en el plano coronal, torsión de la columna vertebral y del tronco y trastorno en el perfil sagital. Esta alteración postural se evalúa mediante radiografía anteroposterior, utilizando el método de Cobb. El presente estudio tiene como objetivo verificar la influencia de la experiencia del evaluador para la fiabilidad intraexaminador e interexaminador del ángulo de Cobb en las curvaturas escolióticas de los niños. El estudio incluyó a 39 niños con escoliosis idiopática entre 7 y 18 años de edad. Los exámenes fueron evaluados por dos fisioterapeutas, un quiropráctico y un estudiante de fisioterapia, siendo que cada uno evaluó cada examen dos veces. Tras siete días, ocurrió una segunda evaluación para la fiabilidad intraexaminador. Además, las primeras evaluaciones proporcionaron datos para la fiabilidad interexaminador. El análisis estadístico se realizó con el coeficiente de correlación intraclase (ICC), con el análisis de Bland y Altman y con el análisis descriptivo de la desviación media absoluta, del error estándar de medición y del cambio mínimo detectable. Se observó una alta fiabilidad (ICC>0,5) en los análisis intraexaminadores entre los profesionales, y una baja fiabilidad (ICC=0,4) en los de evaluadores inexpertos. La fiabilidad interexaminador de los profesionales fue buena $(I C C=0,6)$, y la presencia del evaluador inexperto fue baja $(I C C=0,3)$. Las evaluaciones entre los profesionales mostraron una menor variabilidad de las medidas y valores de desviación estándar en comparación con los del evaluador inexperto. La medición de los ángulos de escoliosis utilizando el método de Cobb que había sido realizada por profesionales con experiencia mostró mejores índices de concordancia y fiabilidad intra e interexaminadores y una menor desviación estándar y variabilidad entre las mediciones. Palablas clave | Columna Vertebral; Rayos X; Reproducibilidad de los Resultados.

\section{INTRODUÇÃO}

A escoliose é definida como uma deformidade com desvio lateral da coluna no plano coronal, torsão da coluna e do tronco, bem como distúrbios no perfil sagital ${ }^{1}$. Dentre sua tipologia, cita-se a escoliose idiopática, congênita, neuromuscular e degenerativa, cada uma predominante em determinada faixa etária e com peculiaridades que as diferenciam e as caracterizam. A escoliose idiopática adolescente (AIS), sem causa conhecida, é a forma mais comumente encontrada em crianças saudáveis; é definida como uma curvatura maior do que $10^{\circ}$, de acordo com o ângulo de Cobb, tendo prevalência de $2 \%$ a $3 \%$. A prevalência de curvaturas maiores do que $20^{\circ}$ é entre $0,3 \%$ e $0,5 \%$, enquanto curvaturas maiores de $40^{\circ} \mathrm{Cobb}$ são encontradas em menos de $0,1 \%$ da população ${ }^{2}$. O diagnóstico e conduta tardios podem resultar em deformidades sérias, afetando a aparência física, função cardiopulmonar e bem estar psicológico.

A avaliação dessa alteração postural é realizada por meio de radiografia de incidência anteroposterior (AP), utilizando-se o método de Cobb - recomendado pela
Scoliosis Research Society, considerado padrão ouro para a mensuração das escolioses ${ }^{3}$ e usado para tomada de decisões sobre a progressão da curvatura e sucesso no tratamento ${ }^{4}$. Essa técnica mede a amplitude da curva pela mensuração e cálculo do ângulo entre linhas, respectivamente traçadas, que tangenciam a placa terminal superior da vértebra cranial e a placa inferior da vértebra caudal da curva escoliótica a ser medida ${ }^{5,6}$. Alguns fatores interferem nessa avaliação, como: seleção da vértebra terminal, tanto caudal quanto cranialmente pertencentes à curva; marcadores radiográficos largos; mensuração de diferentes observadores; inexatos transferidores; erro da construção da técnica e design; erro no desenho da perpendicularidade ou do próprio ângulo ${ }^{4}$.

Cinco graus de progressão já são considerados clinicamente relevantes em muitos estudos. No entanto, a variabilidade intra e interexaminador na determinação do ângulo Cobb em radiografias apresenta diferenças de $4^{\circ}$ a $8^{\circ}$, respectivamente ${ }^{7-9}$. Embora haja, teoricamente, algumas vantagens na técnica, já tem sido demonstrada pouca confiabilidade interexaminador na seleção das últimas vértebras para avaliação das escolioses ${ }^{10}$. 
Além dessas dificuldades quando se pretende mensurar o ângulo Cobb em crianças, essa avaliação se torna mais complexa por elas apresentarem imaturidade óssea, ossificação incompleta e desenvolvimento anômalo da vértebra terminal, dificultando o traçado de uma linha reta sobre o platô vertebral para formação do ângulo ${ }^{11}$. Tais dificuldades, somadas à inexperiência do avaliador na avaliação radiológica, parecem ser fatores que contribuem para a variabilidade angular encontrada nos estudos, mas até o momento não se conhece a real interferência da experiência do avaliador nessas avaliações. Dessa forma, o objetivo deste estudo foi verificar a influência da experiência do avaliador sobre a confiabilidade intra e interexaminador das medições do ângulo Cobb em curvaturas da coluna vertebral de crianças com escoliose.

\section{METODOLOGIA}

Foram avaliadas radiografias panorâmicas frontais da coluna vertebral de 39 pacientes portadores de escoliose idiopática, com idade compreendida entre 7 e 18 anos, que realizaram esse exame em um hospital de Porto Alegre durante o período de realização do estudo. Foram encontradas oito curvas em "S", sendo somente a curvatura principal analisada, e, nos demais sujeitos, curva única. Para a obtenção dos exames, os pacientes não foram submetidos a nenhuma carga de radiação além daquela já necessária para o seu acompanhamento ambulatorial.

As radiografias foram examinadas por quatro avaliadores (A, B, C e D). Cada um avaliou duas vezes cada exame, sendo a segunda avaliação após sete dias, caracterizando a confiabilidade intraexaminador. Além disso, a confiabilidade obtida entre as primeiras avaliações forneceu os dados das análises interexaminadores. As avaliações foram realizadas utilizando o software Matlab 7.9, com base no método descrito por $\mathrm{Cobb}^{12}$, e de forma independente; ou seja, cada avaliador necessitava definir as vértebras a serem marcadas, e não havia conhecimentos dos resultados obtidos pelos demais avaliadores. Foram excluídos pacientes submetidos a alguma cirurgia na coluna vertebral, aqueles com alguma anomalia congênita e aqueles com radiografias que apresentavam características não compatíveis com escoliose idiopática.

Dos quatro avaliadores, três eram profissionais com um mínimo de 5 anos de experiência na área de ortopedia, acostumados a analisarem radiografias da coluna vertebral -, sendo dois fisioterapeutas e um quiropraxista (avaliadores A, B e C). Um deles era estudante de Fisioterapia (avaliador D), que estava no quinto semestre do curso. Previamente à realização das avaliações, todos realizaram um treinamento de três horas, o qual consistiu na análise de radiografias utilizando o software Matlab 7.9. Além disso, os pacientes somente foram incluídos após concordarem em participar do estudo e após a assinatura do Termo de Consentimento Livre Esclarecido por seus pais ou responsáveis.

Para fins de análise, inicialmente foi verificada a normalidade dos dados por meio do teste de ShapiroWilk. Os dados foram analisados utilizando o coeficiente de correlação intraclasse (CCI) e a análise de Bland e Altman. Também realizamos uma análise descritiva, com média, desvio-padrão (DP), cálculo do desvio absoluto médio (DAM), mínima mudança detectável (MMD) e medida do erro-padrão da medição (EPM). O CCI foi classificado de acordo com Fleiss ${ }^{13}$ como baixo, quando se obteve $\mathrm{CCI}<0,4$; bom, quando o CCI estava entre 0,4 e 0,75; e excelente, quando $\mathrm{CCI}>0,75$. Adotou-se o nível de significância de 0,05 em todos os testes, sendo todas as análises estatísticas executadas no software SPSS versão 17.0.

\section{RESULTADOS}

A amostra foi composta por 39 radiografias, sendo a idade média dos pacientes de 12,94 $\pm 3,03$ anos, massa corporal de $48,52 \pm 13,93 \mathrm{~kg}$, estatura de $1,50 \pm 0,15 \mathrm{~m} \mathrm{e}$ IMC de $21,08 \pm 3,84 \mathrm{~kg} / \mathrm{m}^{2}$. A Tabela 1 apresenta os valores de média e desvio-padrão (DP) de cada avaliador no $1^{\circ}$ e $2^{\circ}$ dia de avaliação. Pode-se constatar que o avaliador $\mathrm{D}$ possui maiores valores de $\mathrm{DP}$ em relação aos demais.

Tabela 1. Média e desvio-padrão (DP) do ângulo Cobb para escoliose nas medições dos quatro avaliadores (A, B, C e D)

\begin{tabular}{|c|c|c|}
\hline Escoliose $(n=39)$ & $\begin{array}{l}1^{\circ} \text { avaliação } \\
\text { Média (DP) }\end{array}$ & $\begin{array}{l}2^{\circ} \text { avaliação } \\
\text { Média (DP) }\end{array}$ \\
\hline Avaliador A & $10,4+3,4^{\circ}$ & $10,8+3,7^{\circ}$ \\
\hline Avaliador B & $11,9+4,4^{\circ}$ & $12,0+4,6^{\circ}$ \\
\hline Avaliador C & $10,5+4,2^{\circ}$ & $9,8+4,3^{\circ}$ \\
\hline Avaliador D (inexperiente) & $13,8+7,6^{0}$ & $13,2+7,3^{\circ}$ \\
\hline
\end{tabular}

\section{Confiabilidade intraexaminador}

Foram observadas confiabilidades alta, demonstradas por CCI maiores que 0,553 (Tabela 2) entre os profissionais (avaliadores A, B e C), enquanto o CCI relativo ao avaliador $\mathrm{D}$ indicou uma confiabilidade baixa. Em relação ao EPM e MMD, os valores giraram em torno de $2,3^{\circ}$ e $4,6^{\circ}$, respectivamente, nas medidas dos 
profissionais, enquanto o avaliador $\mathrm{D}$ apresentou valores significativamente maiores. O cálculo do DAM de cada avaliador nas medidas de confiabilidade intraexaminador mostrou valores similares entre eles, porém o desvio-padrão verificado no DAM do avaliador $\mathrm{D}$ foi significativamente maior do que o dos demais.

Tabela 2. Resultados de confiabilidade intraexaminador (avaliadores A, B, C e D) da medição do ângulo de Cobb para escoliose

\begin{tabular}{|c|c|c|c|c|c|}
\hline $\begin{array}{l}\text { Escoliose } \\
(n=39)\end{array}$ & $\begin{array}{c}\mathrm{CCl} \\
\text { (IC95\%) }\end{array}$ & $\mathbf{P}$ & EPM & MMD & DAM \\
\hline Avaliador A & $\begin{array}{r}0,553 \\
(0,292-0,738)\end{array}$ & $<0,001^{*}$ & $2,4^{\circ}$ & $4,7^{\circ}$ & $-0,3 \pm 3,4^{\circ}$ \\
\hline Avaliador B & $\begin{array}{r}0,711 \\
(0,512-0,837)\end{array}$ & $<0,001^{*}$ & $2,4^{\circ}$ & $4,7^{\circ}$ & $-0,04 \pm 3,4^{\circ}$ \\
\hline Avaliador C & $\begin{array}{r}0,735 \\
(0,550-0,852)\end{array}$ & $<0,001^{*}$ & $2,1^{\circ}$ & $4,2^{\circ}$ & $0,6 \pm 3,0^{\circ}$ \\
\hline $\begin{array}{l}\text { Avaliador D } \\
\text { (inexperiente) }\end{array}$ & $\begin{array}{r}0,451 \\
(0,162-0,669)\end{array}$ & $0,002^{*}$ & $5,3^{\circ}$ & $10,5^{\circ}$ & $0,5 \pm 7,8^{\circ}$ \\
\hline
\end{tabular}

${ }^{*}$ Confiabilidade significativa; CCl: coeficiente de correlação intraclasse; EPM: erro-padrão da medição; MMD: mínima mudança detectável; DAM: desvio absoluto médio.

\section{Confiabilidade interexaminador}

A avaliação da confiabilidade interexaminador (Tabela 3) foi realizada com os valores das primeiras avaliações de cada um. Foi observada confiabilidade alta entre os profissionais (avaliadores A, B e C), enquanto o $\mathrm{CCI}$ referente ao avaliador $\mathrm{D}$ indicou uma confiabilidade baixa. Em relação aos valores de EPM e MMD, foram verificados maiores erros de medidas quando a análise foi realizada com as medições do avaliador D.

Tabela 3. Resultados da confiabilidade interexaminador das medições do ângulo de Cobb para escoliose entre os avaliadores A, B, C e D (este último corresponde ao avaliador inexperiente)

\begin{tabular}{|c|c|c|c|c|c|}
\hline $\begin{array}{l}\text { Escoliose } \\
(n=39)\end{array}$ & $\begin{array}{c}\mathrm{CCl} \\
(\mathrm{IC} 95 \%)\end{array}$ & $p$ & EPM & MMD & DAM \\
\hline $\begin{array}{l}\text { Todos } \\
\text { avaliadores }\end{array}$ & $\begin{array}{r}0,298 \\
(0,141-0,483)\end{array}$ & $<0,001^{*}$ & $4,1^{\circ}$ & $8,1^{\circ}$ & $3,8 \pm 3,7^{\circ}$ \\
\hline $\begin{array}{l}\text { Avaliadores } \\
\mathrm{A}, \mathrm{B} \text { e C }\end{array}$ & $\begin{array}{r}0,648 \\
(0,485-0,781)\end{array}$ & $<0,001^{*}$ & $2,4^{\circ}$ & $3,8^{\circ}$ & $2,9 \pm 2,90$ \\
\hline
\end{tabular}

*Confiabilidade significativa. CCl: coeficiente de correlação intraclasse; EPM: erro-padrão da medição; MMD: mínima mudança detectável; DAM: desvio absoluto médio

\section{Concordância interexaminador}

A concordância entre os avaliadores (Tabela 4) $(\mathrm{A} \times \mathrm{B}$; $\mathrm{A} \times \mathrm{C} ; \mathrm{A} \times \mathrm{D} ; \mathrm{B} \times \mathrm{C} ; \mathrm{B} \times \mathrm{D}$ e $\mathrm{C} \times \mathrm{D})$ foi calculada por meio das análises de Bland e Altman, usando os valores das primeiras avaliações. Para as análises com o estudante (D) é possível observar média das diferenças mais distante de zero e maiores desvios-padrão, consequentemente, maiores limites superior e inferior, em relação aos profissionais (A, B e C).
Tabela 4. Resultados das Análises de Bland Altman para identificação das concordâncias entre todos os avaliadores (A, B, C e D, sendo este último correspondente ao avaliador inexperiente)

$\begin{array}{cccccc}\text { Avaliadores } & \begin{array}{c}\text { Média das } \\ \text { diferenças } \\ (\stackrel{\circ}{)}\end{array} & \begin{array}{c}\text { Limite } \\ \text { superior } \\ (\stackrel{\circ}{)})\end{array} & \begin{array}{c}\text { Limite } \\ \text { inferior } \\ (\stackrel{\circ}{)}\end{array} & \begin{array}{c}\text { Desvio } \\ - \text { padrão } \\ (\stackrel{\circ}{)}\end{array} & \begin{array}{c}\text { Indivíduos } \\ \text { fora dos } \\ \text { limites }\end{array} \\ \mathrm{A} \times \mathrm{B} & -0,69 & 6,71 & -8,08 & 3,77 & 1 \\ \mathrm{~A} \times \mathrm{C} & -0,01 & 6,64 & -6,67 & 3,40 & 0 \\ \mathrm{~A} \times \mathrm{D} & -1,37 & 14,88 & -17,62 & 8,29 & 1 \\ \mathrm{~B} \times \mathrm{C} & 0,67 & 7,59 & -6,24 & 3,53 & 1 \\ \mathrm{~B} \times \mathrm{D} & -0,68 & 16,74 & -18,11 & 8,89 & 0 \\ \mathrm{C} \times \mathrm{D} & -1,36 & 14,73 & -17,45 & 8,21 & 0\end{array}$

\section{DISCUSSÃO}

No que tange à avaliação da confiabilidade intraexaminador, este estudo apresentou menor CCI e maiores DP em suas medidas, caracterizando uma avaliação com maior variabilidade. Possivelmente, esse resultado exprime o fato de que o avaliador $\mathrm{D}$ não tem tanta experiência e não está tão familiarizado com a técnica quanto os demais, sendo um indicativo de que a experiência profissional pode ser um importante fator para a adequada utilização do método de Cobb na avaliação da escoliose.

$\mathrm{Na}$ avaliação da confiabilidade interexaminador com as medidas de todos os avaliadores, encontrou-se baixa confiabilidade. No entanto, quando se considerou somente as avaliações dos profissionais, encontrou-se confiabilidade alta e significativa, o que suporta a hipótese inicial de que a experiência do avaliador pode ser um fator que explica os diferentes níveis de confiabilidade intra e interexaminadores encontrados nos estudos que utilizam o método de Cobb.

Em relação à concordância interexaminador, destacamos que no nosso estudo as avalições entre os profissionais possuem valores mais aproximados de zero para a média das diferenças e menor variabilidade entre as medidas, considerando os DP e os limites superior e inferior. Resultados contrários aos nossos foram encontrados por Ritter et al. ${ }^{14}$, nos quais a baixa concordância, tanto para os avaliadores experientes quanto para os inexperientes, foi atribuída às dificuldades de avaliação do método, e não devido à experiência da pessoa. Não obstante, ainda são escassos os estudos que analisam a concordância entre avaliadores considerando a experiência profissional.

$\mathrm{Na}$ literatura, os estudos sobre confiabilidade ainda são muitos divergentes. Por exemplo, Lenke et al. ${ }^{15}$ encontraram alta confiabilidade tanto intra quanto interexaminadores, demonstrada por valores de Kappa 
de 0,92 e 0,83 respectivamente; Ogon et al. ${ }^{16}$ encontraram valores de 0,73 e 0,62 para as mesmas variáveis. Uma possível explicação para essa variabilidade entre os estudos pode ser a experiência e, por consequência, o julgamento do profissional que está fazendo a medição. Isso porque a seleção das vértebras terminais pertencentes à curva enquadra-se como importante parâmetro para mensuração da curva escoliótica idiopática ${ }^{5}$, sendo que, quando as vértebras são previamente determinadas, o erro é menor ${ }^{4}$. Concordando com isso, tem sido demonstrado que o método de Cobb pode estar propenso a maior erro em virtude da seleção de diferentes vértebras terminais ${ }^{6} \mathrm{e}$ da estimativa de inclinações diferentes das mesmas ${ }^{6,17}$. $\mathrm{E}$, justamente por isso, avaliadores inexperientes poderiam apresentar maior dificuldade nessa escolha e consequentemente maior variabilidade em suas medidas.

O grande objetivo de calcular os valores de CCI está no fato de se analisar os valores de EPM e MMD, já que essas variáveis apresentam os valores de erro que são inerentes à medida em si. Neste estudo, os avaliadores experientes tiveram valores de acordo com os relatados na literatura ${ }^{18}$. Porém, quando foram adicionados os valores referentes ao inexperiente, houve um erro significativamente maior em todos os aspectos de confiabilidade, tanto intra quanto interexaminador, o que reforça a importância da experiência do profissional ao realizar as mensurações, já que isso possibilita uma análise mais precisa, com menor erro entre as medidas.

Este estudo encontrou variabilidade de $-0,3^{\circ}$ a $0,6^{\circ}$ para a confiabilidade intraexaminador entre os profissionais (Tabela 2), valores menores do que o encontrado no estudo de Ylikoski e Tallroth ${ }^{19}$, de 4,6 $6^{\circ}$ Quando se analisou a variabilidade intraexaminador do avaliador $\mathrm{D}$, o valor dessa medida não aumentou muito; porém, o seu desvio-padrão foi substancialmente maior do que os encontrados entre os demais avaliadores, indicando que avaliação é menos confiável se realizada por alguém inexperiente. Apesar disso, a literatura também apresenta maiores valores de variabilidade para essa medida como, por exemplo, $9,6^{\circ 11}$ e $10,4^{\circ 7}$, apesar de não relatarem inexperiência do avaliador.

Considerando que os tratamentos para escoliose são determinados com base na medida angular do desvio da coluna vertebral ${ }^{7}$, entende-se que é importante conhecer de forma precisa a variabilidade inerente a esse exame, uma vez que, na prática clínica, uma variação superior a $5^{\circ}$ indica uma progressão da curvatura ${ }^{6}$. Dado esse contexto, aconselha-se que os avaliadores das radiografias, no que tange à avaliação da escoliose, sejam treinados no método. Tradicionalmente, nas situações clínicas, essa já é uma prática comum. No entanto, no que se refere ao acompanhamento de pacientes portadores de escoliose idiopática, muitas vezes os exames são analisados por diferentes profissionais, o que não corresponde à situação ideal, já que a literatura aponta maiores valores de confiabilidade interexaminador em relação à intraexaminador ${ }^{3}$. Não obstante, no ambiente da pesquisa, muitas vezes a avaliação da escoliose pelo método Cobb é realizada pelos próprios pesquisadores, nem sempre treinados no método, o que pode justificar as divergências encontradas.

\section{CONCLUSÃO}

As medições do ângulo Cobb em curvaturas escolióticas de crianças sofreram influência da experiência do avaliador, pois as medidas realizadas pelos profissionais experientes apresentaram melhores índices de concordância, bem como de confiabilidade intra e interexaminador e menor variabilidade, quando comparadas às medidas do avaliador inexperiente.

\section{REFERÊNCIAS}

1. Weiss HR, Negrini S, Rigo M, Kotwicki T, Hawes MC, Grivas $T B$, et al. Indications for conservative management of scoliosis (guidelines). Scoliosis Spinal Disord. 2006;1(1):1. doi: 10.1186/1748-7161-1-5

2. Weinstein SL. Natural history. Spine. 1999;24(24):2592-600.

3. Rosenfeldt MP, Harding IJ, Hauptfleisch JT, Fairbank JT. A comparison of traditional protractor versus Oxford Cobbometer radiographic measurement: intraobserver measurement variability for Cobb angles. Spine. 2005;30(4):440-3. doi: 10.1097/01.brs.0000153401.78638.cb

4. Gstoettner M, Sekyra K, Walochnik N, Winter P, Wachter, R, Bach CM. Inter- and intraobserver reliability assessment of the Cobb angle: manual versus digital measurement tools. Eur Spine J. 2007;16:1587-92. doi: 10.1007/s00586-007-0401-3

5. Mehta SS, Modi HN, Srinivasalu S, Chen T, Suh SW, Yang $\mathrm{JH}$, Song HR. Interobserver and intraobserver reliability of Cobb angle measurement: endplate versus pedicle as bony landmarks for measurement: a statistical analysis. J Pediatr Orthop. 2009;29(7):749-54. doi: 10.1007/s00247-009-1464-6

6. Zhang J, Lou E, Le LH, Hill DL, Raso JV, Wang Y. Automatic Cobb measurement of scoliosis based on fuzzy Hough Transform with vertebral shape prior. J Digit Imaging. 2009;22(5):463-72. doi: 10.1007/s10278-008-9127-y

7. Carman DL, Browne RH, Birch JG. Measurement of scoliosis and kyphosis radiographs. Intraobserver and interobserver variation. J Bone Joint Surg Am. 1990;72(3):328-33. 
8. Geijer H, Beckman KW, Jonsson B, Andersson T, Persliden J. Digital radiography of scoliosis with a scanning method: initial evaluation. Radiology. 2001;218(2):402-10. doi: 10.1148/ radiology.218.2.r01ja32402

9. Gross C, Gross M, Kuschner S. Error anlaysis of scoliosis curvature measurement. Bull Hosp Jt Dis Orthop Inst. 1983;43(20):171-7.

10. Potter BK, Rosner MK, Lehman Jr RA, Polly Jr DW, Schroeder TM, Kuklo TR. Reliability of end, neutral and stable vertebrae identification in adolescent idiopathic scoliosis. Spine. 2005;30:1658-63. doi: 10.1097/01.brs.0000170290.05381.9a

11. Loder RT, Urquhart A, Steen H, Graziano G, Hensinger RN, Schlesinger A, et al. Variability in Cobb angle measurements in children with congenital scoliosis. J Bone Joint Surg Br. 1995;77(5):768-70.

12. Cobb J. Outline for the study of scoliosis. Instr Course Lect. 1948;5:261-75.

13. Fleiss JL. The design and analysis of clinical experiments. New York: John Wiley \& Sons; 1986.

14. Ritter R, Nagasse Y, Ribeiro I, Yamazato C, Oliveira FMD, Kusabara R. Comparison of Cobb angle measurement in scoliosis by residents and spine experts. Coluna/Columna. 2016;15(1):13-6. doi: 10.1590/S1808-185120161501147274

15. Lenke LG, Betz RR, Harms J, Bridwell KH, Clements DH, Lowe TG, et al. Adolescent idiopathic scoliosis: a new classification to determine extent of spinal arthrodesis. J Bone Joint Surg Am. 2001;83(8):1169-81. doi: 10.2106/00004623-200108000-00006

16. Ogon M, Giesinger K, Behensky H, Wimmer C, Nogler M, Bach CM, et al. Interobserver and intraobserver reliability of Lenke's new scoliosis classification system. Spine. 2002;27(8):858-62.

17. Allen S, Parent E, Khorasani M, Hill DL, Lou E, Raso JV. Validity and reliability of active shape models for the estimation of cobb angle in patients with adolescent idiopathic scoliosis. J Digit Imaging. 2008;21(2):208-18. doi: 10.1007/s10278-007-9026-7

18. Troyanovich SJ, Harrison DE, Harrison DD, Holland B, Janik TJ. Further analysis of the reliability of the posterior tangent lateral lumbar radiographic mensuration procedure: Concurrent validity of computer- aided X-ray digitization. J Manipulative Physiol Ther. 1998;21(7):460-7.

19. Ylikoski M, Tallroth K. Measurement variations in scoliotic angle, vertebral rotation, vertebral body height, and intervertebral disc space height. J Spinal Disord. 1990;3(4):387-91. 